\title{
Fill the gap: A novel test to elicit nominal plurals in Sign Language of the Netherlands
}

\author{
Cindy van Boven (ACLC, University of Amsterdam) \\ c.m.j.vanboven@uva.nl
}

\begin{abstract}
The present study introduces a novel gap-filling test to elicit plural nouns in Sign Language of the Netherlands (NGT). As of yet, nominal plurals in NGT have not been described in detail, as eliciting plural nouns is not without challenges. In previous research on NGT (Zwitserlood and Nijhof 1999), native signers were asked to describe pictures of plural objects. However, when describing pictures, the signers automatically also expressed the spatial distribution of the objects depicted on the stimulus picture, using localization. As a consequence, it remains unclear what 'pure' plurals - without localization - look like. The goal of our gap-filling task is to disentangle pluralization from localization: participants are asked to insert plural nouns in signed sentence contexts where the spatial distribution of the referents is irrelevant. After piloting the task, five deaf native signers participated. The task succeeded in eliciting pure plural forms that were not spatially distributed, and the results show that NGT optionally employs reduplication to mark the pure plural of nouns. We conclude that our gap-filling task successfully controls for localization, targeting the desired structure without using written language. In future studies, the gap-filling task can be applied to other sign languages, targeting also other construction types.
\end{abstract}

Keywords: gap-filling task, data elicitation, methodology, nominal pluralization, Sign Language of the Netherlands

\section{Introduction}

Elicitation-based methods are valuable for collecting sign language data for linguistic research. However, sign language data elicitation comes with some specific challenges. For instance, while spoken language elicitation typically relies on native speakers, accessing native signers to participate in a study is not straightforward: due to the varying sociolinguistic settings in which sign languages are acquired, native signers usually are scarce (e.g., Costello, Fernández, and Landa 2008). Moreover, it is important to design appropriate elicitation materials: Tasks that involve translation from spoken (written) language should be avoided, since we cannot assume all signers know the spoken language well enough, and if they do, translating will likely influence their signed responses (Fischer 2009). Elicitation material should therefore be completely visual, e.g., picture and/or video stimuli (Fischer 2009; Padden 2015; for more discussion of several aspects of sign language data collection, see also e.g. Nyst 2015; Quer and Steinbach 2019).

The present study addresses the issue of designing an elicitation task that does without written language and still is successful in targeting the desired structure. More specifically, 
we develop a suitable methodology to elicit nominal plurals in Sign Language of the Netherlands (Nederlandse Gebarentaal; NGT). We discuss previous research on this topic in the following section.

\subsection{Eliciting nominal plurals in a sign language}

In their study on pluralization in NGT, Zwitserlood and Nijhof (1999) elicited plural nouns by asking native signers to describe pictures of singular and plural entities, in different spatial arrangements: 22 pictures showed plural objects arranged neatly, while 20 pictures showed plural objects in a random arrangement. ${ }^{1}$ The picture stimuli were pre-tested with three hearing, Dutch-speaking participants, ensuring that the pictures actually elicited singular and plural forms. Indeed, speakers of Dutch described pictures of objects in different spatial arrangements by simply pluralizing the noun. It turned out, however, that this is not the case for NGT signers: they formed the plural not through marking on the noun, but rather by using modality-specific strategies for localizing entities, such as classifiers, contour signs, and pointing signs in space. The locations where these signs are articulated are meaningful, as they indicate the spatial arrangements of the objects shown on the picture stimuli. Thus, for instance, when shown a picture of bicycles arranged neatly in a row, the NGT noun BICYCLE was followed by a classifier handshape referring to the bicycle (a vertically oriented flat hand), which was repeated and spatially distributed to indicate that spatial arrangement, as shown in Figure 1 (Zwitserlood and Nijhof 1999: 69).

Figure 1: Pluralization by means of localizing classifier handshapes in space (Zwitserlood and Nijhof 1999: 69; (C UiL OTS; reprinted with permission).

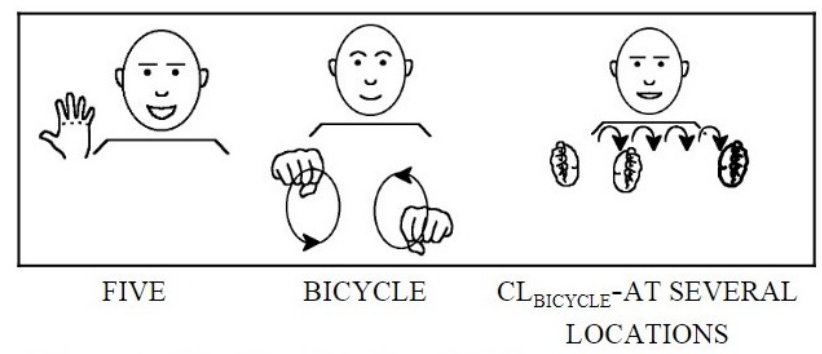

There are five bicycles (in a line).

Thus, when presented with a picture of bicycles, signers do not simply express the plural form 'bicycles', but rather describe how exactly these bicycles are depicted on the picture: 'Five bicycles in a line'. The close relation between localization and pluralization is certainly interesting, as it turns out to play an important role in describing multiple entities in specific spatial arrangements in NGT.

Strikingly, Zwitserlood and Nijhof (1999) find that reduplication ${ }^{2}$ of the noun sign is not a systematic pluralization process in NGT, even though reduplication has been reported as a dominant pluralization strategy for many other sign languages (e.g., Pfau and Steinbach 2005). Indeed, nominal reduplication may not apply when signers describe the spatial configuration of the plural objects. However, as a consequence of the chosen methodology, i.e.,

\footnotetext{
${ }^{1}$ Note that a similar picture-description task to elicit plurals was also used for Turkish Sign Language by Zwitserlood, Perniss, and Özyürek (2012).

${ }^{2}$ Under reduplication, a (part of the) stem is repeated in order to form a new meaning - in this case, the plural.
} 
a picture description task, the study cannot provide information on 'pure' plural forms - that is, plural forms where the spatial arrangement of the objects does not play a role, such as 'bicycles' in a sentence like 'He stole the bicycles'.

Harder, Koolhof, and Schermer (2003) also looked into nominal pluralization in NGT, using a different methodology: they did not elicit data, but rather analyzed more spontaneous signing, namely teaching materials for students of NGT that contain plural nouns (importantly, the topic of the selected materials was not pluralization). Again, the authors find that in some cases, the referents are localized in space. Yet, unlike Zwitserlood and Nijhof (1999), they also find that nominal reduplication - that is, reduplicating the noun sign without the implication of spatial distribution - systematically occurs. While this study does not explicitly distinguish 'pure' plurals from localized plurals, it at least suggests that localization of classifiers and pointing signs is not the only pluralization strategy in NGT, and that nominal reduplication may play a role.

\subsection{Research aims}

As of yet, it is still unclear which pluralization strategies are used to mark 'pure' plural nouns in NGT. Clearly, when describing pictures, signers tend to convey more information than the plural alone. Therefore, our aim is to develop an alternative and more suitable methodology for eliciting plural nouns: a novel gap-filling task, which allows us to disentangle spatial distribution from pluralization. Besides this methodological contribution - which we hope will be used with other sign languages in future studies - we also present the first results from conducting this task, offering new insights into how plurality is marked in NGT.

The following section details how the gap-filling task was designed and conducted. Then, Section 3 presents the first results of the task, and argues that the task succeeded in disentangling pluralization from spatial distribution. The section concludes with some methodological challenges. Finally, Section 4 draws a conclusion.

\section{A novel gap-filling task to disentangle pluralization from distribution in NGT}

\subsection{Design}

Our goal was to design a task that elicits plural nouns without using written Dutch, i.e., using only visual and signed material, to minimize the influence of the surrounding spoken language. At the same time, it was clear that we could not use picture stimuli alone, as this would likely present us with the same pitfalls as discussed above. Hence, in order to elicit pure plurals that do not convey a specific spatial arrangement, we designed a novel gapfilling task.

Gap-filling tasks in written form have previously been used in spoken language research. For instance, Engberg-Pedersen and Boeg Thomsen (2016) developed such a task to test the acquisition of Danish particles by autistic children. Moreover, a comparable methodology is the so-called "cloze test", often used to measure language proficiency and reading skills, first presented by Taylor (1953), but see also, among many others, Potter (1968); Oller and Conrad (1971); and Tremblay (2011). A cloze test has also been used to investigate reading ability in deaf individuals (Reynolds 1986). 
Our task is not written but signed, and presents participants with stimuli that consist of two elements: (i) a signed sentence in which one sign (the target plural noun) is omitted and replaced by a question mark sign - importantly, the spatial configuration of the referents is irrelevant in these sentences; (ii) a picture presented simultaneously with the sentence, showing an illustration of the target plural noun. Signers are instructed in NGT, by a deaf native signer, to repeat the sentence, and replace the question mark sign by the sign for the objects they see in the picture. The idea is that signers will modify the noun such that it is pluralized, and fits into the sentence context.

First, we decided which nouns should be included in the task. Because previous research (e.g., Pfau and Steinbach 2005 on German Sign Language) has shown that phonological features of the base noun (i.e., place of articulation and movement type) can influence the choice of pluralization strategy, we took into account these properties. That is, we designed carrier sentences for 21 nouns with different phonological specifications: six non-body-anchored simple movement nouns (place of articulation lateral or midsagittal), nine non-body-anchored complex movement nouns (repeated, circular, and/or alternating movement), ${ }^{3}$ and six body-anchored nouns. Moreover, since previous research (e.g., Pfau and Steinbach 2005) also suggested that pluralization of a noun may be blocked by the cooccurrence of numerals and quantifiers, we designed two carrier sentences for each noun: one containing a numeral or quantifier, and one without. Thus, in total, 42 sentences were created for 21 different nouns.

The sentence contexts were designed such that spatial configuration is completely irrelevant; that is, the contexts mostly involve non-specific, generic referents (such as farmers, babies, or people in general) and/or verbs that do not imply a specific spatial arrangement of their arguments (such as 'to buy' or 'to have'). When designing the contexts, a deaf native NGT signer was consulted. In (1), we provide examples of carrier sentences without $(1 \mathrm{a}, 1 \mathrm{c})$ and with ( $1 \mathrm{~b}, 1 \mathrm{~d})$ numerals and quantifiers.
(a) LAST OCTOBER [FARMER] STRIKE
'Last October, [the farmers] were on strike.'
(b) MANY [FARMER] TRACTOR HAVE
'Many [farmers] have a tractor.'
(c) INDEX BABYSIT PERSON $_{1}$. OFTEN [CHILD] PLAY
'I am a babysitter. I often play with [children].'
(d) MY SISTER THREE [CHILD] HAVE
'My sister has three [children].'

The sentences are presented by a deaf native NGT signer. She signs the complete sentences, except for the plural nouns (indicated between brackets in (1)); these are omitted, and replaced by the index finger tracing the shape of a question mark in space. The omitted plural referents are presented by means of photos and illustrations, simultaneously with the signed sentence. The referents appear in varying spatial arrangements; sometimes in a row, sometimes distributed more randomly - we expected that this would not matter, as spatial configuration is irrelevant in the sentence contexts. Figure 2 shows the item for the sentence in (1 a), and Figure 3 shows the item for the sentence in ( $1 \mathrm{~d})$.

\footnotetext{
${ }^{3}$ In order to include all types of complex movement nouns, i.e. with repeated, circular, and/or alternating movement, more complex movement nouns were included than simple movement nouns and body-anchored nouns.
} 
Figure 2: Carrier sentence and picture stimulus presented in the gap-filling task, cf. ( $1 \mathrm{a}$ ). Targeted answer: LAST OCTOBER FARMER(PL) STRIKE. ${ }^{4}$

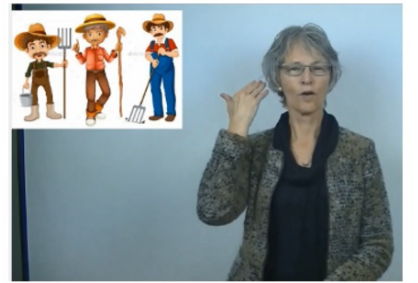

LAST

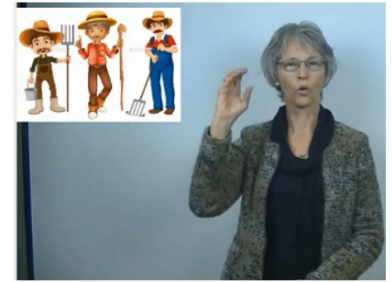

OCTOBER

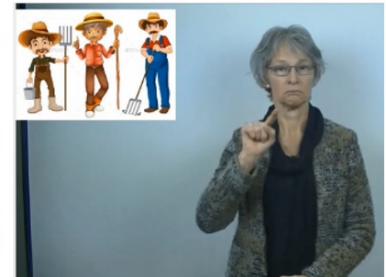

QUESTION-MARK

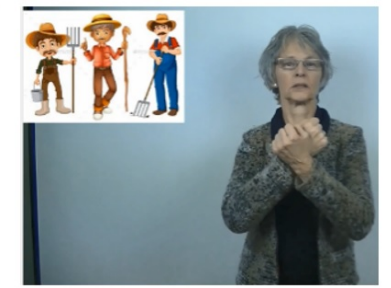

STRIKE

Figure 3: Carrier sentence and picture stimulus presented in the gap-filling task, cf. $(1 \mathrm{~d})$. Targeted answer: MY SISTER THREE CHILD(PL) HAVE ${ }^{5}$

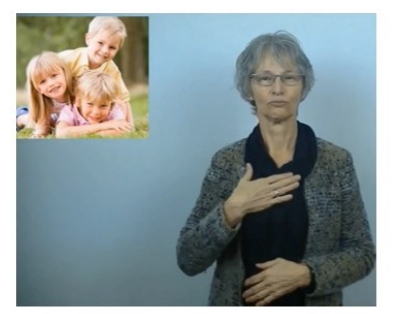

MY

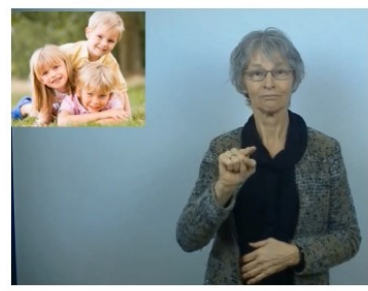

QUESTION-MARK

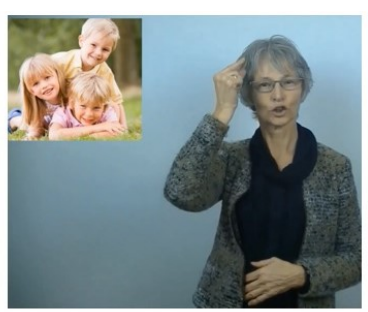

SISTER

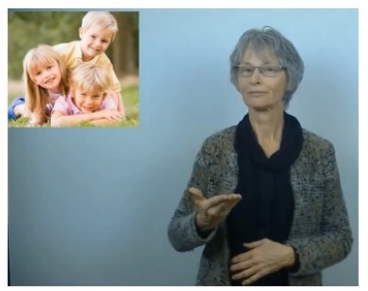

HAVE

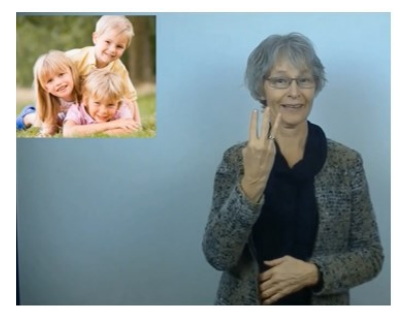

THREE

Moreover, we added 11 sentences designed in exactly the same way, in which, however, a singular noun was omitted rather than a plural noun. These sentences were included for two reasons: (i) to add some variation to the test, such that participants would not realize we are looking for plurals, and (ii) to provide us with a baseline of nouns that have an inherent repetition in their base form (i.e., the complex movement nouns). For instance, NGT singular nouns such as BICYCLE, TRAIN, and RESTAURANT are lexically specified for a repeated circular and/or alternating movement; however, the exact number of inherent repetitions is subject to individual variation. In order to be able to distinguish inherent repetition from potential plural marking by means of reduplication, we also elicit the singular form of these nouns by means of sentences such as (2). This allows us to compare the number of repetitions within participants.

(2) INDEX $_{1}$ NEW [BICYCLE] BUY

'I bought a new bicycle.'

\footnotetext{
${ }^{4}$ Picture stimulus 'farmers' from https://www.canstockphoto.com/farmers-22036428.html; (C) Can Stock Photo / colematt

${ }^{5}$ Picture stimulus 'children' from https://www.canstockphoto.com/three-young-children-playingoutdoors-1722535.html; (c) Can Stock Photo / monkeybusiness
} 
Thus, in total, participants are presented with 53 carrier sentences. These carrier sentences are presented in a semi-randomized order, which is the same for all participants. ${ }^{6}$ No more than four sentences eliciting a plural noun are presented in a row without a singular carrier sentence intervening. Moreover, no more than two sentences containing a numeral/quantifier are presented in a row. The order is also varied such that nouns with similar phonological specifications do not follow each other, and the same target noun is never presented contiguously.

In order to ensure that these sentences indeed elicit plural nouns, and to confirm that the instructions and task were clear, we first piloted the test with one native signer. She took part in the test as any other participant would, but afterwards, we asked her some specific questions on the test and some of her answers. She confirmed that the test was clear, and her answers also suggested that the carrier sentences indeed elicit 'pure' plurals without spatial configuration, as we had hoped. Before testing more participants, we changed five sentences, either because the pilot participant thought they were not clear, or because they elicited singular forms rather than plural forms. After making the necessary changes, other participants took part in the gap-filling task.

\subsection{Participants}

Excluding the pilot participant, five deaf, native NGT signers (one male, four female) participated. They each filled out a form with some background questions. The age range of the participants is 25-62 years (mean age 38.4 years). Three participants come from a hearing family, while the others have deaf family members. Importantly, all participants grew up with NGT.

\subsection{Procedure}

Participants were tested individually. Before starting the actual task, they saw a video in which a native signer explained the topic of the research in NGT. Participants were not told the exact goal beforehand, however, they were informed that the research is about nouns in different sentence contexts. After the information about the research in general, participants received the instructions for the task, again by a native signer. They were instructed to press 'play' on the laptop in front of them, watch the sentence and the corresponding figure closely, and then press 'pause'. After pressing 'pause', participants were asked to turn towards the camera, and to repeat the sentence without changing anything, except for the question mark sign, which they were instructed to replace with a noun, based on the picture that they saw. When they were finished, they pressed 'play' again to move on to the next carrier sentence. To make sure participants understood what to do, they first saw an example sentence followed by the corresponding answer; this example elicited a singular noun that was not included in the rest of the task. At the end of the instruction, a hearing signer of NGT asked in NGT whether everything was clear and whether participants had any questions. Then the actual task started, and during the entire task, the hearing signer was present to answer any further questions.

\footnotetext{
${ }^{6}$ We are aware that it would have been preferable to vary the order of the test items between participants. Yet, when first using the task, this strategy was not implemented: just one version of the test was created, since only five signers participated. Future users of the gap-filling task - especially in a larger scale experiment are advised to vary the order in which sentences are shown to the different participants, by creating multiple versions of the test.
} 


\subsection{Analysis}

All elicited nouns were annotated in ELAN (Crasborn and Sloetjes 2008). We started by glossing the elicited nouns; usually, the gloss corresponded to the noun we aimed to elicit, but in some cases the participant inserted a non-target noun in the sentence (e.g., FRIEND instead of HUMAN). If the participant produced a different variant of a noun than the one we aimed for, this was also annotated. As long as the noun referred to a plural referent, we included it in our analysis. We excluded instances where participants - despite instructions - changed the sentence context such that a singular rather than a plural noun was inserted; this happened only infrequently. If participants changed the sentence context slightly, but still articulated a plural noun, we included it. For the nouns with plural referents, we indicated the relevant phonological properties (for a complete overview of this phonological analysis, see van Boven n.d.). Finally, we annotated if and how the plural was marked.

\section{Results and discussion}

\subsection{Pluralization and localization disentangled}

The gap-filling task succeeded in eliciting pure plural forms that were not spatially distributed. Given that there were 42 test items targeting a plural noun for each of the five participants, in principle we would have expected to elicit 210 plural noun tokens. Yet, 21 tokens were excluded, given the criteria in Section 2.4 - thus, in total, 189 plural noun tokens were elicited.

The results show that, indeed, NGT has morphological strategies to express plurality, without localizing the referents in the signing space. That is, these strategies apply to nouns in sentence contexts where the exact spatial distribution of the real-world items is irrelevant. The different strategies and their distributions are given in Table 1.

Table 1: Elicited pluralization strategies.

\begin{tabular}{|c|c|}
\hline Pluralization strategy & Number \\
\hline Zero marking & 72 \\
\hline Simple reduplication (Figure 4) & 71 \\
\hline Sideward reduplication (Figure 5) & 44 \\
\hline Others & 2 \\
\hline Total & 189 \\
\hline
\end{tabular}

Table 1 shows that 61.9 percent of the elicited nouns (i.e., 117 out of 189) is morphologically marked for plural. If plurality is marked on the noun sign in NGT, this can be done by two types of reduplication: simple reduplication - i.e., repeating the noun at one location - illustrated in Figure 4, and sideward reduplication - i.e., repeating the noun while moving the hand sideward - illustrated in Figure $5 .^{7}$ Note that these strategies are not obligatory: the other 38.1 percent is zero marked. Finally, the presence of numerals or quantifiers in the sentence does not systematically block reduplication.

\footnotetext{
${ }^{7}$ We indicate simple reduplication with a + added to the gloss of the sign; we indicate sideward reduplication with >+ added to the gloss of the sign. In both cases, the number of pluses corresponds to the number of repetitions.
} 
Figure 4: Simple reduplication of CHAIR in NGT.

a.

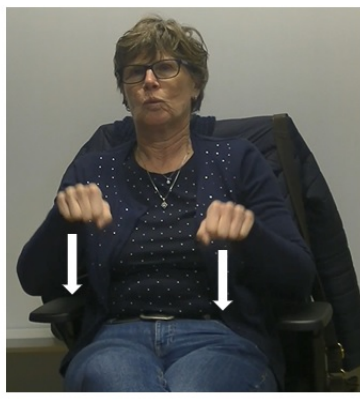

CHAIR
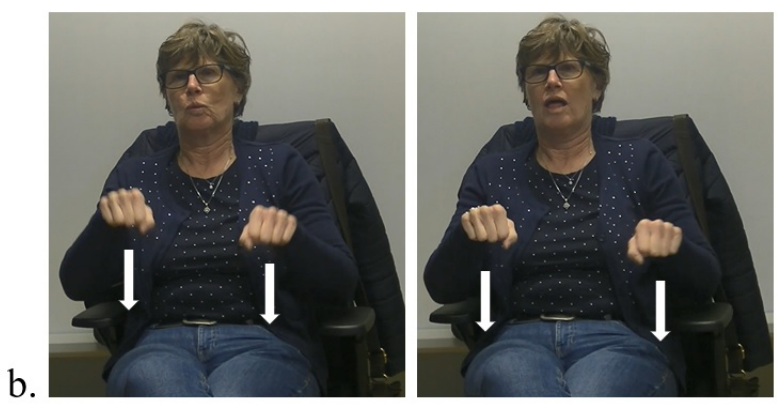

CHAIR+

Figure 5: Sideward reduplication of CHILD in NGT.

a.

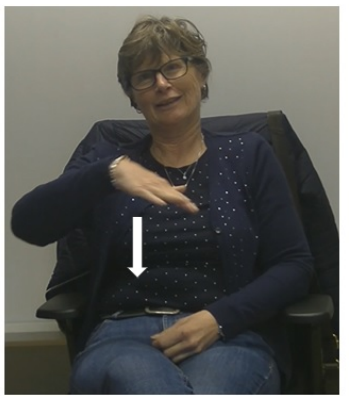

CHILD

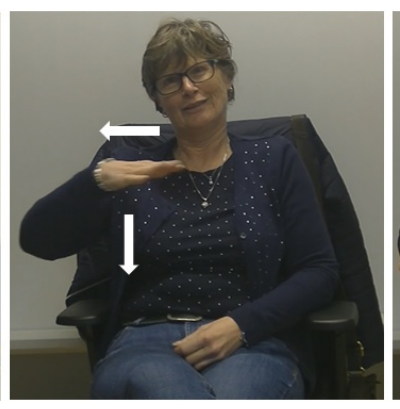

CHILD $>+>+$

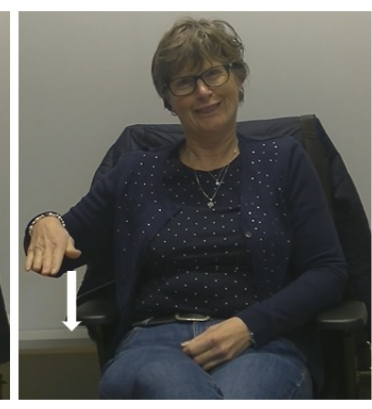

b.

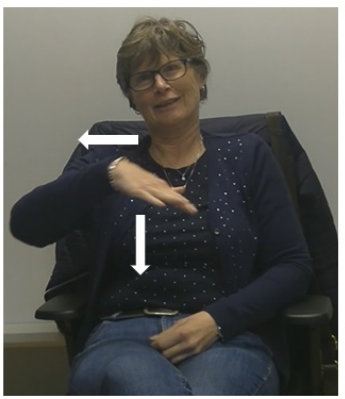

In crucial contrast to Figure 1, the sideward reduplication illustrated in Figure 5 does not localize referents in space: the meaning expressed in Figure 5 is not 'three children next to each other', but rather just 'children' - this becomes clear when we look at the contexts in which CHILD was elicited (1 c, $1 \mathrm{~d}$ ).

Additional evidence that the strategies found here do not reflect the spatial arrangement of the referents comes from the elicitation pictures used: For instance, for elicitation of the plural form of CHILD , signers saw the elicitation picture in Figure 3, repeated below in Figure 6. The children on the picture are not standing in a neat row, but still signers pluralized CHILD by means of sideward reduplication. If we compare Figure 5 to Figure 6 , it is clear that the spatial arrangement did not influence the pluralization strategy.

Figure 6: Picture eliciting the plural form of CHILD.

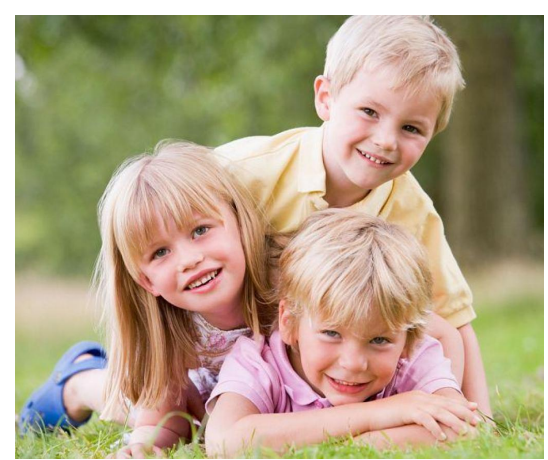


Comparing the results of our gap-filling task to previous research illustrates how findings can differ depending on methodology. Our results suggest that nominal reduplication is a common pluralization strategy, while Zwitserlood and Nijhof (1999) suggest that nominal reduplication does not occur in NGT, because it is barely used when describing pictures of plural entities. This illustrates that, when eliciting sign language data (be it plurals or some other grammatical phenomenon), it is of utmost importance to be aware of the impact of the resources afforded by the visual-spatial modality: if the stimulus picture depicts spatial distribution, then the signer will likely encode this component of meaning in her production. For some grammatical phenomena, this may not pose problems. Yet, if the use of space needs to be controlled for, a gap-filling task offers a promising alternative way to elicit data without using written language. Future research should investigate other domains of sign language grammar in which gap-filling tasks can be fruitfully applied.

Apart from controlling for spatial distribution, we should note one final advantage of data elicitation in general and our gap-filling task in particular: it allows the researcher to control for the specific nouns that are elicited. Remember that we included nouns with varying phonological properties in our test. Indeed, our analysis suggests that the choice between simple and sideward reduplication largely depends on those phonological properties, rather than the spatial distribution of the referents. For instance, as illustrated in Figure 5 above, sideward reduplication typically applies to simple movement nouns articulated at the lateral side of the signing space, such as CHILD in NGT (for more details on phonologically conditioned allomorphy, see van Boven n.d.).

\subsection{Methodological challenges}

Although the gap-filling task worked as intended, there are some drawbacks that we do not want to withhold from the reader. First, when signers carried out the task, it turned out to be quite unnatural for them to exactly repeat a given sentence, and only fill in the gap without making any other changes. There appear to be at least two causes for this. The first is that NGT shows quite some variation: regional lexical variation (Schermer 2004), as well as syntactic variation that manifests itself, for instance, in a - to some extent - flexible word order (e.g., Pfau and Bos 2016). As a consequence, while the carrier sentences were completely natural to the native signer who assisted us in designing the study, some of the participants preferred a different word order, or different variants of some signs. When participants indicated this was the case, the present hearing signer of NGT asked how they would normally sign the sentence. For instance, one carrier sentence that was considered unnatural by multiple signers is INDEX 1 TWO [GLASSES] HAVE, BECAUSE SPARE CONVENIENT ("I have two pairs of [glasses] because a spare pair is convenient"). ${ }^{8}$ Three signers indicated that they were not familiar with the sign SPARE we included in the test, and that they preferred to use a different variant. Additionally, three signers changed the order of the object and the verb in the first half of the sentence. These changes were allowed, as they are not expected to influence the pluralization strategy.

The second reason why signers did not always exactly repeat the sentence, might be more problematic. In some cases, signers did not modify the noun for plurality, but rather some other element in the sentence - often, this was the verb. Thus, they sometimes adapted the carrier sentences in order to mark plurality. This indicates that marking the plural on the noun sign may not always be the preferred strategy, and that in natural discourse, and in certain contexts, there may be a preference for marking plurality on the verb. Obviously,

${ }^{8}$ While the English gloss GLASSES is uderlyingly plural, this is not the case for the NGT sign. 
this is a more general shortcoming of elicitation tasks: the patterns we find may not always reflect the most natural language use. Therefore, it is important to complement this type of data with naturalistic (corpus) data (for advantages and drawbacks of combining corpus data and elicited data, see Kimmelman, Klomp, and Oomen 2018).

A final drawback that should be pointed out concerns the fact that elicitation likely does not provide us with a complete picture of all possible nominal pluralization strategies in the language. First, the use of visual stimuli did not allow us to elicit the plural form of abstract nouns, as these cannot be depicted on a picture for insertion in the carrier sentence. Additional research is thus necessary to come to know whether similar pluralization strategies apply to abstract nouns, for instance, by means of grammaticality judgements. Second, we elicited plurals only in very specific semantic environments - mostly generic, general contexts. This limitation is inherent to the idea behind the task: we were interested in how nouns are pluralized when spatial configuration is irrelevant, and as a consequence, we only looked at these specific contexts. One should bear in mind, however, that a study using this task as its only method does not provide a complete picture of pluralization strategies across all semantic contexts. Hence, in this respect, too, complementing the findings with more naturalistic data is necessary.

\section{Conclusion}

The present study introduces a novel gap-filling task, designed for the elicitation of plural nouns in NGT. The task is a valuable methodological tool, as it is easy to administer and offers us a way to disentangle pluralization from spatial distribution, properties that were shown to be conflated in a previously used picture-description task. Five native NGT signers were presented with signed sentences, from which the target plural noun was omitted and replaced by a question mark sign. They were asked to repeat the sentences, and to fill in the gap. In crucial contrast to elicitation by means of picture stimuli, spatial distribution of the target entities was irrelevant in the elicited sentences. Indeed, the task succeeded in eliciting 'pure' plural forms, that is, plural nouns that are not spatially distributed. Our results suggest that both simple and sideward reduplication are used to pluralize the noun sign in NGT, although these strategies are not obligatory. This clearly shows that results from previous studies - as interesting as they may be - were incomplete, and thus that the methodology, i.e., gap-filling task versus picture description, influences which pluralization strategies are used by the participants.

To conclude, this study shows that a gap-filling task can be fruitfully applied to sign languages, bringing to light new results. Therefore, future research should explore further possibilities for using a gap-filling task to elicit sign language data. We are confident that this type of task can be applied in other linguistic domains in which it is desirable to control for the semantic and/or syntactic environment. Moreover, for spoken languages, gap-filling tasks have been used, for instance, in studies on first and second language acquisition (e.g. Yamashita 2003; Engberg-Pedersen and Boeg Thomsen 2016). Future research should investigate these possibilities for sign languages, too.

\section{Acknowledgements}

I wish to thank Marijke Scheffener for all her help with designing the task; Roland Pfau for his very useful feedback on earlier versions of this paper; and - importantly - I am grateful to all 
the signers who participated in this study. This work is part of the research programme $\mathrm{PhDs}$ in the Humanities with project number PGW19.003, funded by the Dutch Research Council (NWO).

\section{References}

van Boven, Cindy (n.d.). "Phonological restrictions on nominal pluralization in Sign Language of the Netherlands: Evidence from corpus and elicited data". Manuscript submitted for publication.

Costello, Brendan, Javier Fernández, and Alazne Landa (2008). "The non-(existent) native signer: Sign language research in a small population”. In: Sign Languages: Spinning and Unravelling the Past, Present and Future. TISLR 9: Papers from the 9th Theoretical Issues in Sign Language Research Conference, Florianópolis, Brazil, December 2006. Ed. by Ronice Müller de Quadros. Petrópolis/RJ, Brazil: Editora Arara Azul, pp. 77-94.

Crasborn, Onno and Han Sloetjes (2008). "Enhanced ELAN functionality for sign language corpora”. In: Construction and Exploitation of Sign Language Corpora. 3rd Workshop on the Representation and Processing of Sign Languages. Ed. by Onno Crasborn et al. Paris: ELDA, pp. 39-43.

Engberg-Pedersen, Elisabeth and Ditte Boeg Thomsen (2016). "The socio-cognitive foundation of Danish perspective-mixing dialogue particles". In: Viewpoint and the Fabric of Meaning: Form and Use of Viewpoint Tools across Languages and Modalities. Ed. by Barbara Dancygier, Lu Wei-Lun, and Arie Verhagen. Berlin: De Gruyter Mouton, pp. 125-142.

Fischer, Susan (2009). "Sign language field methods: Approaches, techniques, and concerns". In: Taiwan Sign Language and Beyond. Ed. by James H-Y. Tai and Jane Tsay. Chia-Yi, Taiwan: The Taiwan Institute for the Humanities, National Chung Cheng University, pp. 119.

Harder, Rita, Corline Koolhof, and Trude Schermer (2003). Meervoud in de NGT: Verslag van een onderzoek in het kader van OCW subsidie 2003. URL: https : / Www . gebarencentrum . $\mathrm{nl} /$ media/33536/94_file1.pdf (visited on 09/23/2019).

Kimmelman, Vadim, Ulrika Klomp, and Marloes Oomen (2018). "Where methods meet: Combining corpus data and elicitation in sign language research". In: Proceedings of the 8th Workshop on the Representation and Processing of Sign Languages: Involving the Linguistic Community. Ed. by Mayumi Bono et al. Paris: Elra, pp. 95-100.

Nyst, Victoria (2015). “Sign language fieldwork”. In: Research Methods in Sign Language Studies. A Practical Guide. Ed. by Eleni Orfanidou, Bencie Woll, and Gary Morgan. Oxford: Wiley Blackwell, pp. 107-122.

Oller, John W. and Christine A. Conrad (1971). “The cloze technique and ESL proficiency”. In: Language Learning: A Journal of Research in Language Studies 321 (2), pp. 183-194. DOI: 10.1111/j.1467-1770.1971.tb00057.x.

Padden, Carol A. (2015). "Methods of research on sign language grammars". In: Research Methods in Sign Language Studies. A Practical Guide. Ed. by Eleni Orfanidou, Bencie Woll, and Gary Morgan. Oxford: Wiley Blackwell, pp. 141-155.

Pfau, Roland and Heleen F. Bos (2016). "Syntax: Simple sentences". In: The Linguistics of Sign Languages. An introduction. Ed. by Anne E. Baker et al. Amsterdam: John Benjamins, pp. 117-148.

Pfau, Roland and Markus Steinbach (2005). "Plural formation in German Sign Language: Constraints and strategies". In: Gebärdensprachen: Struktur, Erwerb, Verwendung (Lin- 
guistische Berichte Special Issue 15). Ed. by Daniela Happ and Helen Leuninger. Hamburg: Buske, pp. 111-144.

Potter, Thomas C. (1968). A taxonomy of cloze Research, part I: Readability and reading comprehension. URL: https://eric.ed.gov/?id=ED035514 (visited on 08/24/2020).

Quer, Josep and Markus Steinbach (2019). "Handling sign language data: The impact of modality”. In: Frontiers in Psychology 10, p. 483. DOI: 10.3389/fpsyg . 2019.00483.

Reynolds, H.N. (1986). "Performance of deaf college students on a criterion-referenced, modified cloze test of reading comprehension". In: American Annals of the Deaf 131 (5), pp. 361364. DOI: 10.1353/aad.2012.1016.

Schermer, Trude (2004). Lexical variation in Sign Language of the Netherlands. URL: https : //www.gebarencentrum.nl/media/33555/92_file1.pdf (visited on 09/23/2019).

Taylor, Wilson L. (1953). “"Cloze procedure": A new tool for measuring readability”. In: Journalism Quarterly 30, pp. 415-433. DOI: 10.1177/107769905303000401.

Tremblay, Annie (2011). "Proficiency assessment standards in second language acquisition research: "Clozing" the gap". In: Studies in Second Language Acquisition 33, pp. 339-372. DOI: $10.1017 / \mathrm{S} 0272263111000015$.

Yamashita, Junko (2003). "Processes of taking a gap-filling test: Comparison of skilled and less skilled EFL readers”. In: Language Testing 20 (3), pp. 267-293.

Zwitserlood, Inge and Sibylla Nijhof (1999). "Pluralization in Sign Language of the Netherlands (NGT)”. In: OTS Yearbook 1998-1999. Ed. by Jan Don and Ted Sanders. Utrecht: UiL OTS, pp. 58-78.

Zwitserlood, Inge, Pamela Perniss, and Asli Özyürek (2012). “An empirical investigation of expression of multiple entities in Turkish Sign Language (TID): Considering the effects of modality". In: Lingua 122, pp. 1636-1667. 